MSO1-03

\section{Serial protein crystallography in living insect cells}

\author{
J. Mia Rudolph ${ }^{1}$, Robert Schönherr ${ }^{2}$, Lars Redecke ${ }^{3}$ \\ 1. University of Lübeck, Biochemistry \& CFEL, DESY, Hamburg, \\ Germany \\ 2. DESY, Photon Science, Hamburg, Germany \\ 3. University of Lübeck, Biochemistry \& DESY, Lübeck, Germany \\ email: rudolph@biochem.uni-luebeck.de
}

Protein crystals in living cells have been observed in all domains of life [1]. This crystallization approach holds the possibility to grow a huge number of micron-sized protein crystals with comparable properties and of high quality in a short time [2-3]. In order to systematically exploit the potential of in cellulo crystallization in living insect cells for structural biology, we are establishing a streamlined process to rapidly elucidate the structural information of in cellulo crystallized target proteins.

After cloning the gene of interest into baculovirus transfer vectors, the associated recombinant baculoviruses are generated to infect insect cells. Crystal formation is detected at day 3 to 5 after infection. If intracellular crystallization was successful, diffraction data of the isolated in cellulo crystals are collected using serial crystallography approaches either at XFELs or highly brilliant synchrotron sources, depending on the crystal diffraction volume. The thousands of generated diffraction patterns are processed either with CrystFEL or XDS. In our hands, these efforts resulted in the successful crystallization of more than 20 different proteins in living insect cells so far. However, a broader application is currently restricted by two major bottlenecks: Firstly, depending on the recombinant protein, the fraction of crystal containing cells varies between more than $80 \%$ and less than $1 \%$. And secondly, changes of environmental conditions during cell lysis and crystal purification result in a loss of crystal quality.

To overcome these limitations we recently established techniques for serial diffraction data collection from in cellulo grown crystals directly within living insect cells, using synchrotron and XFEL radiation both at RT and cryo conditions. These innovative approaches avoid crystal purification and transfer of the living, crystal-containing cells, and furthermore allow direct screening of cell cultures for successful in cellulo protein crystallization using the X-ray beam. Ultimately, our current in cellulo crystallization pipeline will be further improved to overcome limitations in data collection due to low intracellular crystallization efficiency, supported by an additional integration of fluorescence-based cell sorting, enriching the proportion of crystal containing cells. Our results pave the way to a more efficient use of crystal containing insect cells as suitable targets for serial diffraction data collection at synchrotrons and XFELs in the future.

Keywords: serial crystallography, in cellulo, SFX

\section{MS01-04}

\section{Nanobeam precession-assisted 3D electron diffraction reveals a new lysozyme polymorph}

Arianna Lanza ${ }^{1}$, Valentina Cappello ${ }^{1}$, Eleonora Margheritis ${ }^{1}$, Enrico

Mugnaioli ${ }^{1}$, Mauro Gemmi ${ }^{1}$, Gianpiero Garau ${ }^{1}$

1. Center for Nanotechnology Innovation, Istituto Italiano di Tecnologia , Pisa, Italy

email: arianna.lanza@iit.it

Besides the well-established X-ray diffraction, the structural investigation of biological macromolecules can rely on new recently developed techniques. Among these, serial crystallography at X-FELs, single particle cryo-electron microscopy and electron diffraction (ED) allow overcoming the challenges posed by large biomolecules, namely (i) the necessity of preserving hydration, (ii) the difficulty to grow large and well-ordered crystals and (iii) the challenge posed by their high beam sensitivity. ED is particularly promising, because electron microscopes are much more readily available than X-FELs and diffraction methods normally allow higher resolution than imaging methods. Nevertheless, applications of 3D ED to structural biology had not been pursued until very recently, when the feasibility was demonstrated on 3D (sub)micro crystals of test-case protein samples.[1,2]

So far, plunge-frozen macromolecular crystals have been investigated in selected area electron diffraction (SAED) mode while the sample was continuously rotated in order to speed the acquisition time and collect a comprehensive data set before the crystal deteriorates due to beam damage. In this contribution we present the first application of nanobeam precession-assisted 3D ED to biological macromolecules. The advantages of this approach with respect to typical SAED methods employed so far are: (i) the reduction of illuminated sample area, (ii) the possibility to track crystal position during sample tilt and (iii) the chance to get multiple data sets from the same crystal.[3]

Our ED data collection strategy has been successfully applied on the well-known hen egg white lysozyme (HEWL), and allowed us to identify a new monoclinic polymorph that crystallizes only in form of micrometric acicular crystals. The crystal structure of this HEWL polymorph could be solved by molecular replacement, making it the first unknown complete protein crystal structure ever solved by ED.

Keywords: Electron diffraction, proteins, nanocrystals 